\title{
Deformation of a Ferrofluid Droplet in a Simple Shear Flow Under the Effect of a Constant Magnetic field
}

\author{
Paolo Capobianchi, Marcello Lappa, Mónica S. N. Oliveira
}

James Weir Fluid Laboratory, Department of Mechanical and Aerospace Engineering, University of Strathclyde, 75 Montrose Street, Glasgow, G1 1XJ, UK

email: paolo.capobianchi@strath.ac.uk, marcello.lappa@strath.ac.uk, monica.oliveira@strath.ac.uk

\begin{abstract}
In the present work, we investigate the dynamics of a droplet of ferrofluid placed in a shear flow field subjected to the additional action produced by the application of a magnetic field in a direction perpendicular to the flow. The problem is solved in the framework of a moving-boundary method based on the solution of the Navier-Stokes equations complemented with the additional equations required for the determination of the magnetic force. The results reveal interesting changes in the trends displayed by the droplet deformation and inclination angle as a function of the capillary number when the intensity of the magnetic field is varied while maintaining flow conditions corresponding to the Stokes regime. The mechanism of droplet relaxation from equilibrium when the magnetic force is suddenly removed is also investigated. According to our numerical experiments the deformation evolves in time following a harmonic decaying process, which, in the limit of small capillary number, i.e. for very small deformations, can be fairly well represented by the temporal evolution of a simple damped harmonic oscillator.
\end{abstract}

\section{Introduction}

By definition ferrofluids are stable colloidal suspensions of magnetic nanoparticles typically coated with a surfactant dispersed in a non-magnetic carrier liquid (Rosensweig, 1985). These special liquids have the ability to display the same fluidity and viscosity of common substances (such as water or silicone oil) and, at the same time, exhibit magnetic properties typical of solid or liquid metals. By virtue of this peculiar behaviour, ferrofluids have applications in a variety of fields, especially those where non-invasive methods for the control of the considered liquid are a necessary pre-requisite, (e.g. Xia et al., 2010 and references therein). The underlying philosophy or strategy in these cases consists of populating the region of the fluid physical domain that needs to be manipulated with magnetic nanoparticles so that it becomes sensitive to the presence of an external magnetic field.

From a fundamental point of view, related study (paradigm) models reduce to flows involving layers, sheets, jets, liquid bridges, bubbles and droplets. Each of these cases involves the presence of interfaces, which, in turn, imply the existence of discontinuities of physical properties (such as viscosity, density, electric properties (see e.g. Behjatian and Esmaeeli, 2013) and, as in the present case, other parameters or constants being related to the magnetic behaviour). All of these jumps can 
produce specific forces, which are key aspects relating to the practical exploitation of these special fluids.

Apart from practical applications (which we will discuss later on in this introduction), these subjects continue to attract interest due to their fascinating implications in the formulation of a general theory for the behaviour of non-linear systems and related pattern formation processes. As a relevant example showing the richness of phenomena potentially enabled in ferrofluids, it is known that when some critical thresholds are exceeded, the above-mentioned forces can give rise to the so-called Rosensweig instability, which occurs when an initially flat interface is subjected to a static perpendicular magnetic field. This instability typically leads to the emergence of a fascinating hexagonal array of peaks (see, e.g., Chen et al., 2008). Similar effects can be produced by the socalled labyrinthine bifurcation, responsible for the formation of highly branched structures when a droplet of ferrofluid is confined in a Hele-Shaw cell (Dickstein et al., 1993).

Droplets are of special interest in such contexts given the paradigmatic role they play in a multitude of companion fields. Indeed, a variety of standard industrial mixing and separation systems involve two or more immiscible fluids subjected to different types of forces in which discrete fluid bodies (namely droplets) may deform, migrate, collide, coalesce, or breakup due to various effects and instabilities. Along these lines, engineers are often faced with the problem of either separating a liquid dispersion into its components (e.g., solvent extraction systems, see Zander et al., 1990) or making such a dispersion stable for a long period of time (as in the case of emulsions, see, e.g., Ivanov and Kralchevsky, 1997; and of polymer blends, see, e.g., Roths et al., 2002). Droplets are also relevant to a variety of biomedical applications. Microrobots in the form of autonomous or externally controlled droplets, in particular, are of great interest as they have the potential to revolutionize many aspects of medicine (there is some general consensus that these untethered microscopic devices will make existing therapeutic procedures less invasive and will enable new procedures never before possible, Nelson et. al, 2010; Tran and Webster, 2010).

All these studies are also relevant in the context of nanotechnologies and related products. Indeed, self-assembly and related concepts of "deterministic hydrodynamics" have gained increased interest in recent years due to their inherent applicability to new methods for the construction of heterogeneous systems made of multiple component types in nano- and micro-scales (relevant to Labon-a-Chip techniques and microfluidic systems, see, e.g. Morris and Parviz, 2006; Abgrall; Gué, 2007; Chen et al., 2009 and Nguyen 2011).

Due to the contactless control methods enabled accordingly, droplets made of ferrofluids are attracting significant interest as new archetypal systems for the improvement of some of the processes discussed above or simply for the definition and exploration of new paths of fundamental research (see, e.g. Hong et al., 2007; Korlie et al., 2008; and references therein). In particular, most of existing studies on ferrofluid droplets have concentrated primarily on their equilibrium shape and stability under the effect of magnetic fields with different properties (often on the basis of a mathematical 
"analogue", namely, a dielectric droplet in a uniform electric field, see, e.g., Bacri and Salin, 1982; Sherwood, 1988 and Stone et al., 1999; Lavrova et al., 2004; Zhu et al., 2011). It is known that if a ferrofluid drop is assumed to be initially spherical, the application of the magnetic field can produce a mismatch in the normal stress condition at the interface (Drozdova et al. 1979) with the resulting force inducing the drop to elongate in the same direction of the imposed field.

A relevant effort towards the identification of the response of such droplets to the simultaneous presence of a magnetic field and external force fields such as those traditionally at play in the dynamics of dispersions and emulsions has been provided recently by Ki (2010) and Shi et al. (2014), who have considered the coupling between the presence of the magnetic field and the standard droplet sedimentation process induced by gravity. In particular, the effects of the magnetic Bond number, susceptibility, Weber number, Reynolds number, and magnetic field direction on the motion and deformation of the droplet have been investigated. Their results show that the increase in magnetic Bond number or susceptibility can produce larger deformation of the droplet while for high Weber numbers, even the formation of a teardrop-like droplet and an oblate elliptical-cap droplet can become possible by properly orienting the magnetic field.

We further expand this line of inquiry by addressing the interplay between the application of an external magnetic field, such as those assumed in the earlier works cited above, and the presence of another standard force or effect traditionally considered in the study of dispersions or emulsions, namely an imposed shear stress. It is known that fluid drops suspended in another immiscible fluid undergo deformation (and subsequently might breakup into smaller droplets by means of the so-called end-pinching phenomenon) when the bulk fluid is subjected to strong shear flows. Despite the extensive amount of literature on this subject (Rallison, 1984; Stone, 1994; Tucker and Moldenaers, 2002; Bławzdziewicz et al., 2003; Cristini et al., 2003; Barai and Mandal, 2016; Ioannou et al. 2016 and references in all these works), to the best of our knowledge no studies have addressed yet the interplay between the application of a shear flow and of a magnetic field to a ferrofluid.

In this work, we focus on configurations involving a single drop of ferrofluid in a non-magnetic matrix. The problem is approached in the framework of a coupled VOF-Level Set method as discussed in detail in the next section.

\section{Mathematical Formulation}

\subsection{Governing Equations}

Let us consider a system of two immiscible isothermal and incompressible Newtonian fluids in which at least one of the phases is a non-electrically conducting ferrofluid subjected to the influence of a constant magnetic field. In the framework of the so-called "single-fluid" approach (see, e.g., Lappa 2005) the related flow can be described by the continuity and Navier-Stokes equations, which can be cast in compact vector form as 
$\rho\left(\frac{\partial \mathbf{u}}{\partial t}+\mathbf{u} \cdot \nabla \mathbf{u}\right)=-\nabla p+\nabla \cdot(2 \eta \mathbf{D})+\mathbf{F}_{\sigma}+\mathbf{F}_{H}$

where $\mathbf{u}$ is the velocity, $p$ is the pressure and $\mathbf{D}=\frac{1}{2}\left(\nabla \mathbf{u}+(\nabla \mathbf{u})^{\mathrm{T}}\right)$ is the rate-of-strain tensor, $\rho$ and $\eta$ being the density and viscosity of the fluid, respectively. Moreover, the two terms appearing at the right hand side of Eq. (2) account for the presence of the forces driving the considered dynamics, namely surface tension and magnetic effects. The former can be represented mathematically as $\mathbf{F}_{\sigma}=\sigma k \mathbf{n} \delta$ (Brackbill et al., 1992), where $\sigma$ is the interfacial tension, $k$ and $\mathbf{n}$ are the curvature and unit vector perpendicular to the interface, respectively, and $\delta$ is a distribution function taking unit value at the interface and assuming zero value elsewhere. The magnetic force is expressed as $\mathbf{F}_{H}=\mu_{0}(\mathbf{M} \cdot \nabla) \mathbf{H},\left(\right.$ Rosensweig, 1985), where $\mu_{0}$ denotes the permeability of the vacuum, $\mathbf{H}$ is the magnetic field and $\mathbf{M}$ is the magnetization. The gravitational contribution is neglected as we focus on neutrally-buoyant systems. Closure of the model from a mathematical point of view, however, requires additional equations. The so-called magnetostatic Maxwell's equations for a non-conducting ferrofluid read:

$$
\nabla \cdot \mathbf{B}=0
$$

$$
\nabla \times \mathbf{H}=0
$$

with $\mathbf{B}$ being the magnetic induction, which is related to the magnetic field by a specific constitutive relationship:

$$
\mathbf{B}= \begin{cases}\mu_{0}(\mathbf{H}+\mathbf{M}) & \text { in the ferrofluid phase } \\ \mu_{0} \mathbf{H} & \text { in the surrounding phase }\end{cases}
$$

The magnetization, $\mathbf{M}$, can be assumed to be proportional to the magnetic field via the magnetic susceptibility $\chi(\mathbf{M}=\chi \mathbf{H})$. Introducing the magnetic permeability of the ferrofluid phase, $\mu=\mu_{0}(1+\chi)$, and substituting the expression given for $\mathbf{M}$ into Eq. (5), the magnetic induction, $\mathbf{B}$, for the ferrofluid phase can be finally written as $\mathbf{B}=\mu_{0}(1+\chi) \mathbf{H}=\mu \mathbf{H}$.

As by virtue of eq. (4) it is possible to introduce a magnetic scalar potential $\psi$ such that $\mathbf{H}=-\nabla \psi$ ; consideration of eq. (3) leads to the following equation

$$
\nabla \cdot(\mu \nabla \psi)=0
$$

by which it is possible to determine $\psi$, and therefore $\mathbf{H}$. 
Most conveniently, the magnetic force can be finally re-cast as

$$
\mathbf{F}_{H}=\nabla \cdot\left(\mu \mathbf{H} \mathbf{H}^{\mathrm{T}}-\frac{1}{2} \mu|\mathbf{H}|^{2} \mathbf{I}\right)
$$

where I represents the identity tensor.

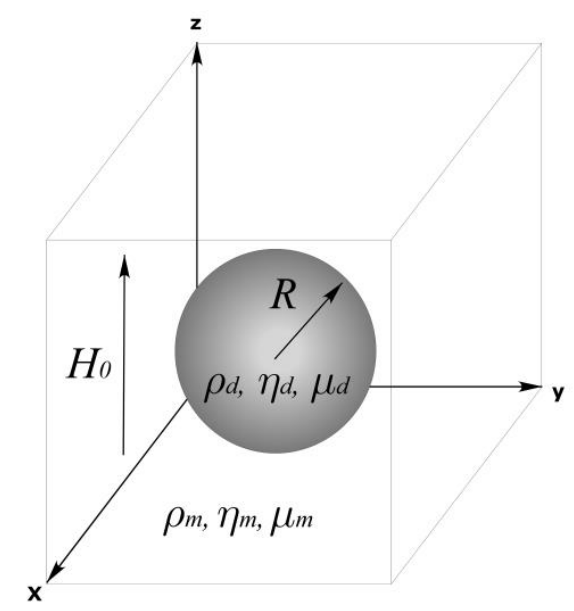

Figure 1: Three-dimensional domain showing a droplet of properties $\rho_{d}, \eta_{d}, \mu_{d}$ surrounded by another immiscible fluid of properties $\rho_{m}, \eta_{m}, \mu_{m}$ in the presence of the magnetic field $H_{0}$.

Assuming as reference quantities, $L_{0}=R, U_{0}$ and $H_{0}$ as length, velocity and magnetic field intensity respectively, $t_{0}=R / U_{0}$ as time scale and $\eta_{m} U_{0} / L_{0}$ as reference for the viscous stress and pressure, the momentum equation can be cast in non-dimensional form as

$\rho_{r}\left(\frac{\partial \mathbf{u}^{*}}{\partial t^{*}}+\mathbf{u}^{*} \cdot \nabla^{*} \mathbf{u}^{*}\right)=\frac{1}{R e}\left(-\nabla^{*} p^{*}+\nabla^{*} \cdot\left(2 \eta_{r} \mathbf{D}^{*}\right)+\frac{1}{C a} \mathbf{F}_{\sigma}^{*}+\xi \mathbf{F}_{H}^{*}\right)$

where the superscript ' $*$ ' denotes dimensionless quantities. The characteristic parameters appearing in eq. (8) are: the Reynolds number, $R e=\rho_{m} U_{0} L_{0} / \eta_{m}$, the capillary number, $C a=\eta_{m} U_{0} / \sigma$ and a parameter measuring the relative importance of magnetic and viscous forces, $\xi=\mu_{m} H_{0}^{2} L_{0} / \eta_{m} U_{0}$ (representing the inverse of the Mason number, $M n$ ). Notice this latter dimensionless quantity is related to the capillary number through the following relationship: $M n=C a / B o_{m}$, where $B o_{m}=\mu_{m} H_{0}^{2} L_{0} / \sigma$ is the magnetic Bond number given by the ratio between the magnetic force and capillary force. Other non-dimensional parameters appearing in the equations are $\rho_{r}=\rho / \rho_{m}$, $\eta_{r}=\eta / \eta_{m}$, (in the remainder we will refer to the matrix (surrounding) and droplet phases using the subscripts " $m$ " and " $d$ ", respectively). Notice that because in the present problem the fluid properties are subjected to a discontinuity across the interface, $\rho, \eta, \chi$ and $\mu$ are formally considered as variables, we will come back to this concept later). 


\subsection{Numerical method}

The required equations for the ferrofluid have been implemented in a multiphase OpenFOAM ${ }^{\circledR}$ solver that relies on a "smoothed" version of a hybrid LS-VOF (Capobianchi et al., 2017a,b) method, which, in turn, is based on the formulation originally introduced by Albadawi et al. (2013) and implemented in OpenFOAM 2.1.x by Yamamoto et al. (2016). In the present section, we describe briefly the main concepts and equations. For further details, the reader is referred to the aforementioned Capobianchi et al. (2017a,b).

In such method, as in the native OpenFOAM solver, the volume fraction $\alpha$ is advected using a surface compression approach:

$$
\frac{\partial \alpha}{\partial t}+\nabla \cdot(\alpha \mathbf{u})+\nabla \cdot\left(\alpha(1-\alpha) \mathbf{u}_{\mathrm{c}}\right)=0
$$

where $\mathbf{u}_{c}$ is an artificial "compressive velocity" (see e.g. Berberovic et al., 2009). The surface compression term, $\nabla \cdot\left(\alpha(1-\alpha) \mathbf{u}_{\mathrm{c}}\right)$, which is effective only in the region across the interface $(0<\alpha<1)$, eliminates the need to reconstruct geometrically the interface and mitigates some wellknown problems due to numerical diffusion. The main advantage of such approach lies in its robustness and capability to handle complex interfaces with a reduced computational cost (see e.g. Klostermann et al., 2012).

The volume fraction $\alpha$ is also used to account for the variability of the material properties over the physical domain, as we formally represent them as $\omega=\alpha \omega_{d}+(1-\alpha) \omega_{m}$ (where $\omega$ is the generic fluid property, e.g., the density $\rho$ or the viscosity $\eta$ ). The magnetic permeability is treated on the same ground, i.e. it is considered as a function of the phase variable $\alpha$ adopting a harmonic interpolation, namely $1 / \mu=\alpha / \mu_{d}+(1-\alpha) / \mu_{m}$ (see e.g Afkhami et al., 2010). This is equivalent to considering the magnetic susceptibility as a piecewise constant (i.e. $\chi=\chi_{d} \neq 0$ in the ferrofluid liquid; and $\chi=\chi_{m}=0$ in the non-magnetizable phase).

Despite the above mentioned benefits, however, these methods can suffer severe problems if "spurious currents" are excited, which generally occur for low capillary number flow regimes. The simplified level set-VOF method introduced by Albadawi et al. (2013) can partially overcome these issues via a specific procedure, as described in the following.

Once the phase function $\alpha$ is calculated by means of Eq. (9), it is possible to define the initial levelset function, $\phi_{0}=(2 \alpha-1) \Delta$, where $\Delta=0.75 \Delta x$ and $\Delta x$ is the grid resolution. Subsequently, a reinitialization equation is solved with the initial condition $\phi(\mathbf{x}, 0)=\phi_{0}(\mathbf{x})$ :

$$
\frac{\partial \phi}{\partial \tau}=\operatorname{sgn}\left(\phi_{0}\right)(1-|\nabla \phi|)
$$


where $\operatorname{sgn}\left(\phi_{0}\right)=\phi_{0} /\left|\phi_{0}\right|$ is the "sign" function (see, e.g., the landmark work by Sussman et al., 1994) and $\tau$ is a fictitious time (the related integration step being fixed to $\Delta \tau=0.1 \Delta x$ ). Subsequently, the level set function $\phi$ is used to evaluate the interface normal and the curvature, required for the calculation of the capillary force $\mathbf{F}_{\sigma}$.

In addition, we introduced a smoothing strategy to further alleviate the occurrence of the aforementioned unphysical parasite currents and improve the geometrical description of the interface. This simple idea is based on the solution of a purely diffusive equation for the level-set function for a prefixed number of cycles $n$ (we have considered $n=2$ for all the computations reported in the present work):

$\phi_{s m}^{n+1}=\phi_{s m}^{n}+\left(\nabla^{2} \phi_{s m}^{n}\right) \Delta t_{f}$

where $\phi_{s m}$ is the "smoothed" level-set function and $t_{f}$ being a fictitious time defined on the basis of stability considerations (the reader is referred again to Capobianchi et al., 2017a,b for a more detailed description of the method) that depends on the grid spacing. Once the smoothed level-set function is known, the normal and curvature at the interface are calculated as

$$
\begin{aligned}
& \mathbf{n}\left(\phi_{s m}\right)=-\frac{\nabla \phi_{s m}}{\left|\nabla \phi_{s m}\right|} \\
& k\left(\phi_{s m}\right)=\nabla \cdot \mathbf{n}\left(\phi_{s m}\right) .
\end{aligned}
$$

We also applied the smoothing strategy to the fluid properties in order to prevent the algorithm from developing spurious oscillations due to the discontinuity at the liquid-liquid interface. More precisely, we have replaced $\alpha$ appearing in the general expressions which account for the variability of $\rho, \eta$ and $\mu$ over the physical domain with the corresponding smoothed version $\alpha_{s m}$ (the "smoothed" volume fraction, $\alpha_{s m}$, being calculated with the same principle used to evaluate $\phi_{s m}$, i.e. by means of an equation analogous to Eq. 11).

Introducing such developments in the momentum equation and considering that one of the terms of the magnetic force is isotropic (we adopt the concept of "corrected pressure", $p_{c}=p+1 / 2|\mathbf{H}|^{2}$ ), we finally obtain

$\rho\left(\frac{\partial \mathbf{u}}{\partial t}+\mathbf{u} \cdot \nabla \mathbf{u}\right)=-\nabla p_{c}+\nabla \cdot(2 \eta \mathbf{D})+\sigma k\left(\phi_{s m}\right) \delta(\phi) \nabla \phi+\nabla \cdot\left(\mu \mathbf{H H}^{\mathrm{T}}\right)$

in which the distribution function $\delta$ is given by

$$
\delta(\phi)= \begin{cases}0 & \text { if }|\phi|>\varepsilon \\ \frac{1}{2 \varepsilon}\left(1+\cos \left(\frac{\phi \pi}{\varepsilon}\right)\right) & \text { if }|\phi| \leq \varepsilon\end{cases}
$$


and $\varepsilon$ is a parameter accounting for the smearing of the interface. Indeed, this parameter is typically used to define the number of cells used for the transition between both fluids. It is obviously a function of the mesh size $(\Delta \mathrm{x})$. In particular, the condition $\varepsilon=1.5 \Delta x$ should be regarded as a "lower limit" to be used to guarantee that surface tension is spread over at least one cell on either side of the interface (Albadawi et al., 2013). Typically, if values larger than this lower limit are used more iterations are required for the solution of the redistancing function as defined by Eq. (10). Some relevant information along these lines can be found in the papers by Sussman et al. (1994); Son and Hur (2002); Sun and Tao (2010). Moreover, when this parameter is increased above the threshold value indicated above (though it has a positive effect on the algorithm in terms of numerical stability), the performances of the method in terms of interface representation accuracy decrease to levels similar to those achievable with a classical VOF method (Albadawi et al., 2013). A relevant procedure for tuning this parameter should consider decreasing its values starting from a given multiple of the minimum allowed value specified above and minimise it verifying that the related changes displayed by the solution are negligible. After testing the sensitivity of our numerical approach to this parameter for the conditions considered in the present work for code validation (Sect. 3.1.2) assuming $\varepsilon=4.5 \Delta \mathrm{x}$, $\varepsilon=3.0 \Delta \mathrm{x}$ and $\varepsilon=1.5 \Delta \mathrm{x}$, we decided to stick to the value suggested by all these authors, i.e. $1.5 \Delta \mathrm{x}$ as the differences between the different solutions for $3.0 \Delta \mathrm{x}$ and $1.5 \Delta \mathrm{x}$ were found to be smaller than $1 \%$.

\section{$\underline{2.3 \text { Solution procedure }}$}

The governing equations have been solved on a structured Cartesian grid using a Finite Volume Method (FVM) relying on an integral formulation of such equations. All the variables are stored at the centre of the cell, however the solution methodology implemented in OpenFOAM also involves their values interpolated at the cell face. In order to avoid unphysical coupling between pressure and velocity (checkerboard effect) due to the non-staggered collocation of the variables, the Rhie-Chow (Rhie and Chow, 1982) interpolation method is used.

The time-marching algorithm for the solution of the entire set of equations can be sketched as follows:

1. Set the boundary and initial conditions;

2. Solve the re-initialization Eq. (10) to calculate the level-set function $\phi$;

3. Solve the diffusion Eq. (11) to get the smoothed level-set function $\phi_{s m}$;

4. Calculate the curvature at the interface by means of Eq. (13);

5. Advect the volume fraction $\alpha$ using Eq. (9) adopting the MULES algorithm (Multidimensional Universal Limiter with Explicit Solution, see e.g. Deshpande et al., 2012). Applying the Gauss theorem, the integration of Eq. (9) leads to

$$
\int_{\Gamma_{c . i}} \frac{\partial \alpha}{\partial t} d V+\int_{\partial \Gamma_{c . i}}\left(\alpha \mathbf{u}+\alpha(1-\alpha) \mathbf{u}_{\mathrm{c}}\right) \cdot \mathbf{n} d S=0
$$


where $\Gamma_{c, i}$ is the volume of the computation cell $i$ and $\partial \Gamma_{c, i}$ its boundary. Using a forward Euler scheme, the discrete counterpart of Eq. (16) can be written as

$$
\left|\Gamma_{c, i}\right| \frac{\alpha_{c, i}^{n+1}-\alpha_{c, i}^{n}}{\Delta t}=-\sum_{f_{c, i}} F_{u}^{n}-\sum_{f_{c, i}} \zeta_{M} F_{c}^{n}
$$

where the flux term $F_{u}$ arises from the integration of $\nabla \cdot(\alpha \mathbf{u})$, and the term $F_{c}$ is a linear combination of the flux associated with the integration of the compressive term $\nabla \cdot\left(\alpha(1-\alpha) \mathbf{u}_{\mathrm{c}}\right)$ and the previous flux $F_{u}$ (see again Deshpande et al., 2012 for more details). The coefficient $\zeta_{M}$ appearing in the second term at the right hand side of Eq. (17) is the MULES limiter. The term $F_{c}$ is active only at the interface, where $\zeta_{M}=1$, while away from the interface $\zeta_{M}=0$ and $F_{c}$ is inactive. The effect of the limiter is therefore to split the numerical treatment of the advection term in two parts: away from the interface the second summation appearing in Eq. (17) is set to zero, and $F_{u}$ is treated with an upwind scheme, while across the interface, where better accuracy is required, a higher order scheme is employed. With this strategy it is possible to reduce the computational effort by enabling the more accurate scheme only in the region of the interface, where higher accuracy is required. Finally, the compressive velocity $\mathbf{u}_{c}$ has the following form

$$
\mathbf{u}_{c}=\min \left[C_{\alpha} \frac{\left|\mathbf{u}_{f} \cdot \mathbf{S}_{f}\right|}{\left|\mathbf{S}_{f}\right|}, \max \left(\frac{\left|\mathbf{u}_{f} \cdot \mathbf{S}_{f}\right|}{\left|\mathbf{S}_{f}\right|}\right)\right] \mathbf{n}_{f}
$$

Here, $\mathbf{u}_{f}, \mathbf{S}_{f}$ and $\mathbf{n}_{f}$ are the values of the velocity interpolated at the cell face, cell face vector (vector normal to the cell face having magnitude equal to its surface area) and cell face normal, respectively. The constant $C_{\alpha}$, is a user defined parameter and is usually set in the range between 0 (i.e. the compressive velocity is inactive) and 2 (larger values of $C_{\alpha}$ lead to a sharper interface but can potentially produce a higher level of spurious currents; All simulations shown in the present work have been performed adopting $C_{\alpha}=2$ ).

6. Solve the equation for the potential (Eq. 6) to get the scalar field $\psi$, which in turn is used to calculate the magnetic field, $\mathbf{H}=-\nabla \psi$;

7. Calculate the magnetic force $\mathbf{F}_{H}$ by means of Eq. (7);

8. Solve the momentum Eq. (14) for a prefixed number of predictor steps to get an initial value of the velocity field;

9. Perform the PISO loop to calculate pressure and velocity fields until momentum and mass conservation are satisfied;

10. Back to step 2 or end of calculation. 


\section{Numerical results}

\section{$\underline{3.1 \text { Validation }}$}

As explained in Sect. 2, the aim of the present work is to study the deformation of a ferrofluid droplet subjected to the combined action of a simple shearing flow and a constant magnetic field applied in a direction perpendicular to the imposed flow. As to the best of our knowledge there are no previous experimental or numerical works addressing such problem, in order to test the accuracy of our numerical implementation, we carried out a series of numerical tests considering the problem of the deformation of (a) a ferrofluid droplet suspended into another quiescent non-magnetizable liquid under the influence of a constant magnetic field without the presence of imposed shear (cf. Section 3.1.1), and (b) a (non-magnetizable) Newtonian droplet in a simple shear flow (cf. Section 3.1.2).

\subsubsection{Ferrofluid droplet in the presence of a magnetic field (no imposed shear flow)}

For the first test case, we performed a set of simulations to mimic a series of experiments by Afkhami et al. (2010), in which a ferromagnetic fluid phase [composed of a colloidal suspension of magnetite $\left(\mathrm{Fe}_{3} \mathrm{O}_{4}\right)$ nanoparticles of average diameter $7.2 \mathrm{~nm}$ dispersed in a PDMS (polydimethylsiloxane) carrier liquid] was suspended in another non-magnetizable Newtonian fluid. In these experiments, the authors placed a small droplet (radius $R=1.291 \mathrm{~mm}$ ) of ferrofluid in a box filled with glycerol ( $\rho_{d}=1260 \mathrm{~kg} / \mathrm{m}^{3}$ ) having previously taken care to minimise the density difference between the two fluids ( $\rho_{m} \cong \rho_{d}$ ) by adjusting the volume fraction of the ferromagnetic particles. The final suspension resulted in a 7 vol. $\%$ of magnetite having a magnetic susceptibility $\chi_{d}=0.8903$. The experimental investigations were carried out by applying different constant magnetic fields. Subsequently the steady state droplet deformation was calculated by image post-processing and the surface tension was deduced on the basis of their theoretical calculations under the hypothesis that the droplet shape could be assumed ellipsoidal. The value obtained for the interfacial tension was $\sigma_{A}=13.5 \mathrm{mN} / \mathrm{m}$. The same theoretical problem was subsequently addressed by Rowghanian et al. (2016), who, without making any assumption on the droplet shape, reported a value of $\sigma_{R}=18.2 \mathrm{mN} / \mathrm{m}$ for the surface tension. For our numerical simulation we adopted both values $\left(\sigma_{A}\right.$ and $\left.\sigma_{R}\right)$ and compared the outcome of the numerical calculations with the experimental measurements.

In particular, our numerical simulations were performed using the geometry adopted by Afkhami et al. (2010), considering a fully three-dimensional parallelepipedic domain of dimensions $L_{r} \times L_{r} \times L_{z}$ ( $L_{r}=7.5 R$ and $L_{z}=15 R$ ), as shown in Fig. 2a. We set no-slip and no-through flow conditions for the velocity at each wall, whilst the presence of the constant magnetic field was introduced by setting a constant value for the magnetic potential, $\psi$, in the two opposite walls perpendicular to the direction of $\mathbf{H}$ (cf. Fig. 2a) in such a way that the resulting magnetic field, $\mathrm{H} \equiv|\mathbf{H}|=-\left(\psi_{z}-\psi_{0}\right) / L_{z}$, was equal to the value used for each of the corresponding experiments (for the sake of simplicity, we have always set $\left.\psi_{0}=0\right)$. We also took advantage of the mesh adaptivity capability offered by the 
OpenFOAM platform by refining the grid only in the region of the droplet in order to reduce the computational effort. A mesh with minimum cell size $R / 50$ was found to be sufficiently refined to provide grid independent results.

Fig. 2b shows the comparison between our numerical findings, the results by Afkhami et al. (2010) and the theory of Rowghanian et al (2016) in terms of droplet aspect ratio measured as the ratio between the major and minor axis of the drop, $\mathrm{b} / \mathrm{a}$. The good agreement is evident for $\mathrm{H}$ up to $4 \mathrm{kA} / \mathrm{m}$ for both values of surface tension considered, but the results deviate on further increasing $\mathrm{H}$. The key to understanding these results lies in the realisation that for small deformations, the assumption of ellipsoidal shape still holds well and both theories provide acceptable results. Remarkably, for higher $\mathrm{H}$, the numerical simulations based on the more accurate surface tension predicted by Rowghanian et al. (2016) are in good agreement with the values obtained experimentally in the whole range of magnetic fields considered.

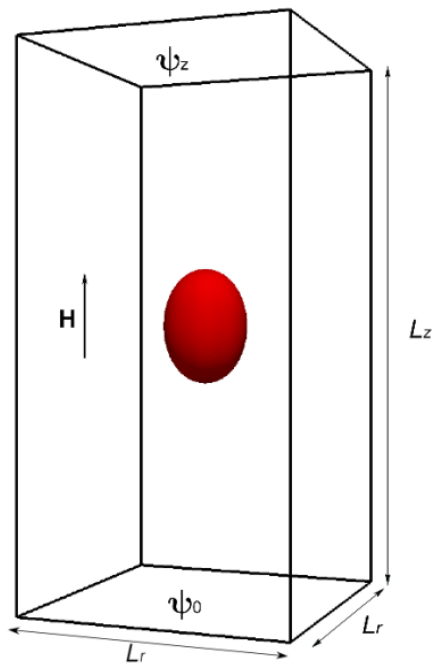

a)

Figure 2: a) Illustration of the domain used for the first test case showing a droplet deformed under the influence of the magnetic field H. b) Droplet aspect ratio b/a for different values of the magnetic field intensity $\mathrm{H}$ : comparison between our numerical simulations (open triangles), the experiments by Afkhami et al. (2010) (open diamonds) and the theory of Rowghanian et al. (2016) (continuous line).

\subsubsection{Non-magnetizable droplet in a simple shear flow (case $\xi=0$ )}

As a second test (before addressing the joint effects of an imposed magnetic field and a simple shearing flow, treated in Sect. 3.2), we have considered the case in which the magnetic field is absent $(\xi=0)$ and inertial effects are negligible $(R e<<1)$ (leading to further validation of the present numerical code through comparison with the existing literature). 
These simulations were carried out considering the benchmark work by Zhou and Pozrikidis, (1993). These authors approached the problem using a boundary integral method (BIM) and considering an initially circular two-dimensional droplet of radius $R$ placed at the centre of a $[\pi L, L]$ domain delimited by two parallel walls moving in opposite directions along the $x$-axis direction with a constant velocity $U_{0}$ (cf. Fig. 3a). As for the initial and boundary conditions, we imposed a constant linear velocity profile along the $x$-direction (including the region occupied by the droplet), while noslip and no-flow through conditions were applied at the walls. The capillary number has been varied by adjusting the surface tension coefficient, keeping the droplet radius and the wall velocity, $U_{0}$ unchanged. The viscosity and density have been assumed to be the same for both fluids.

Fig. $3 \mathrm{~b}$ shows the comparison in terms of deformation parameter, $D=(b-a) /(a+b)$, for different values of the capillary number $\left(C a=\eta_{m} \gamma R / \sigma\right.$, where $\gamma=2 U_{0} / L$ ) of our simulations with the results of Zhou and Pozrikidis (1993); other numerical results (cross symbols) obtained by Yue et al. (2004) using a "diffuse interface method" have also been included in the same plot for the sake of completeness. Our results are in good agreement with other numerical findings in the whole range of $\mathrm{Ca}$ considered. The maximum relative error between our computations and the benchmark data of Zhou and Pozrikidis (1993) is within 5\%. All these results were obtained assuming $\varepsilon=1.5 \Delta \mathrm{x}$. As indicated at the end of Sect. 2.2, we have also assessed the impact of this parameter. As an example, the values taken by $D$ for $C a=0.1$ are 0.1076 and 0.1085 for $\varepsilon=3 \Delta \mathrm{x}$ and $\varepsilon=1.5 \Delta \mathrm{x}$, respectively (percentage variation $<1 \%$ ).

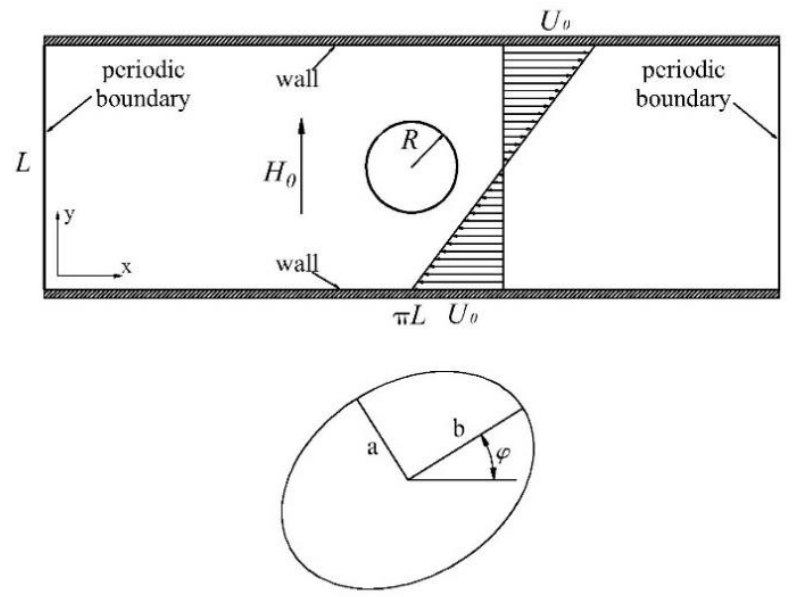

a)

Figure 3: a) Sketch of the domain used for the test cases with simple shear flow (Sect. 3.1.2 and 3.2) (top) and of the deformed droplet showing the minor and major axis and the orientation angle $\varphi$ (bottom) (also showing the considered parameters and the flow conditions; notice the presence of the magnetic field $H_{0}$, which is of relevance to the configuration considered in Sect. 3.2). b) Deformation parameter, $D$, as a function of the capillary number for the case of a non-magnetizable droplet in a simple shear flow: comparison between our results (open triangles) and the simulations by Zhou and Pozrikidis (1993) (open diamonds) and Yue et al. (2004) (cross symbols).

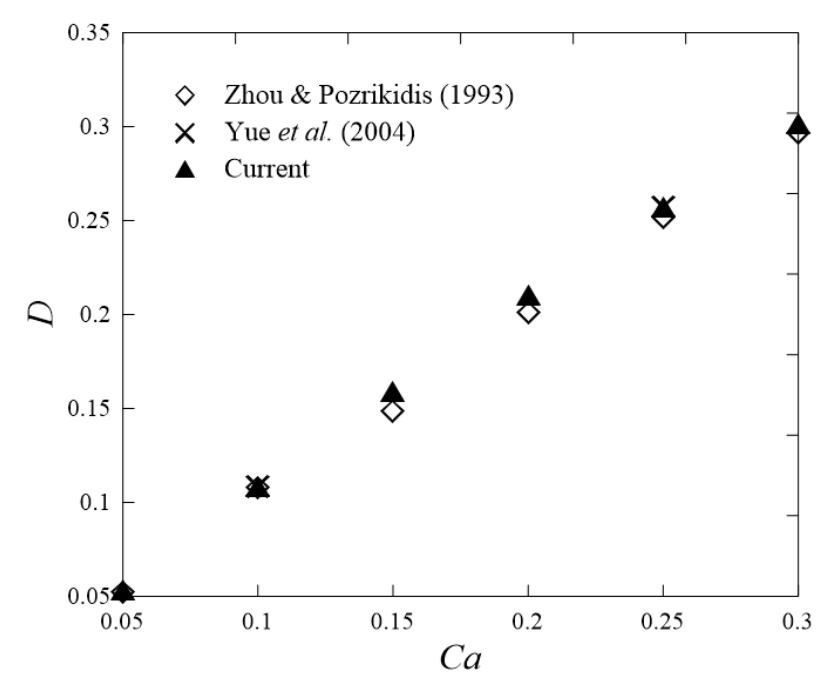

b) 


\subsection{Ferrofluid droplet under combined magnetic field and simple shear flow}

In the present section, we consider the case of a ferrofluid droplet surrounded by another nonmagnetizable fluid subjected to the combined action of a simple shearing flow and a constant magnetic field, $\mathrm{H}_{0} \hat{\boldsymbol{e}}_{y}\left(\hat{\boldsymbol{e}}_{y}\right.$ representing the $y$-axis unit vector) acting perpendicularly to the imposed velocity, $U_{0}$ (cf. Fig. 3a).

The numerical simulations have been conducted considering the same geometrical constrains (domain and confinement) assumed for the problem described in Sect. 3.1.2. The two phases have the same density $\left(\rho_{d}=\rho_{m}\right)$ and the same viscosity $\left(\eta_{d}=\eta_{m}\right)$ defined in such a way that the effect of inertia is negligible $\left(R e=\rho_{m} \gamma R^{2} / \eta_{m} \ll<1\right)$.

Once the viscosity ratio is fixed, the problem can be fully described by the capillary number and the parameter $\xi$ (or alternatively, by the pair $\xi, B o_{m}$ ), and by the value of the magnetic susceptibility of the ferrofluid phase (as susceptibility for the ferrofluid phase we have used the same value adopted for the case shown in Sect. 3.1.1, $\chi_{d}=0.8903$, while in the non-magnetizable phase $\chi_{m}=0$ ). Table 1 shows the values of the fluid properties and other constrains used in the simulations. The capillary number and the parameter $\xi$ were adjusted by varying the values of the interfacial tension and the magnetic field, while maintaining all other parameters unchanged.

Table 1: Fluid properties and other constrains adopted in the simulations considering a ferrofluid droplet under the joint action of simple shear flow and magnetic field

\begin{tabular}{|c|c|c|c|}
\hline \multicolumn{4}{|c|}{ Fluid properties } \\
\hline & $\rho\left[\mathrm{kg} \mathrm{m}^{-3}\right]$ & $\eta[\mathrm{Pa} \mathrm{s}]$ & $\chi[-]$ \\
\hline Droplet & 1 & 0.125 & 0.8903 \\
\hline Matrix & 1 & 0.125 & 0 \\
\hline \multicolumn{4}{|c|}{ Geometric and kinematic constrains } \\
\hline$R[\mathrm{r}$ & & & ${ }_{0}\left[\mathrm{~m} \mathrm{~s}^{-1}\right]$ \\
\hline 0.1 & & & 0.1 \\
\hline
\end{tabular}

It can be seen that under the combined influence of the shear and magnetic force, the fluid particle deforms and orients while still maintaining its position centred in the middle of the domain (no translational motion is produced because the magnetic force is distributed symmetrically around the droplet surface). Figure 4 shows the temporal evolution of the deformation parameter for $\xi=5.65$ and $C a=0.1$ as a function of the dimensionless time, $t^{\prime}=\gamma t$ (product of the actual dimensional time with the imposed shear rate) and for three different maximum Courant numbers, namely $\mathrm{MaxCo}=0.075,0.15,0.3$. We notice that in all the cases the deformation $D$ initially increases, reaches a peak and finally converges to a constant final (steady state) value. Note that for reasons of computational convenience subsequent simulations were carried out setting a maximum Courant 
number of $\mathrm{MaxCo}=0.15$ unless stated otherwise. This value of the maximum Courant number is not sufficiently small to guarantee an accurate evaluation of the transient deformation, but it is adequate to evaluate the steady state deformation in which we are interested in (the difference between the values of $D$ obtained for MaxCo $=0.15$ and for $\mathrm{MaxCo}=0.075$ is less than $0.5 \%$ ).

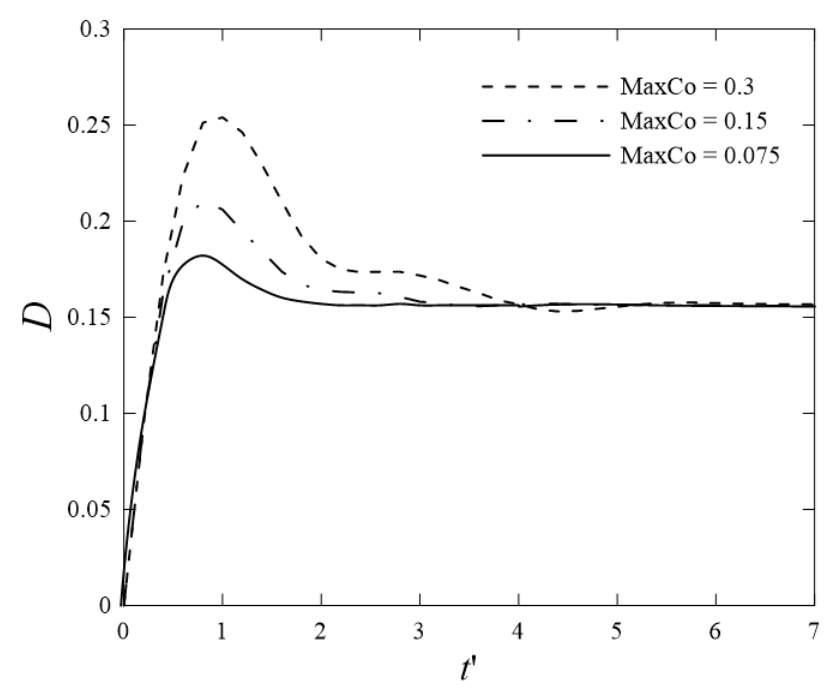

Figure 4: Temporal evolution of the deformation parameter $D$ for different values of the capillary number for a ferrofluid droplet under the joint action of simple shear flow and magnetic field $(\xi=2.51)$.

All the simulations shown here and in the next subsection were carried out using a mesh sufficiently refined to guarantee grid independent results. For the refinement tests, in particular, we considered (for all the capillary numbers and $\xi=5.65$ ) three different degrees of refinement, namely $\mathrm{M}_{1}, \mathrm{M}_{2}$ and $\mathrm{M}_{3}$ corresponding respectively to 45,56 and 70 cells per droplet radius (obtained by increasing the resolution by a factor 1.25 for each refinement step). The results of this study are summarised in Fig. 5 and Table 2. It can be seen that, as the number of cells is increased, the percentage difference displayed by the two sensitive parameters considered for the study, i.e. the steady state deformation $D$ and orientation angle $\varphi$, falls below $2 \%$ as soon as the grid density exceeds that corresponding to the $\mathrm{M}_{2}$ mesh. Accordingly we have used this resolution for all considered cases.

It is worth pointing out that for the simulations presented in Sect. 3.1.2 mesh independence could be achieved with less dense grids. The need for a more refined geometry to resolve the flow problem under discussion might be ascribed to the presence of larger deformations (see the remainder of the present section) and the possible sources of artificial diffusion in the governing equations stemming from the presence of an additional divergence term relating to the magnetic force. 


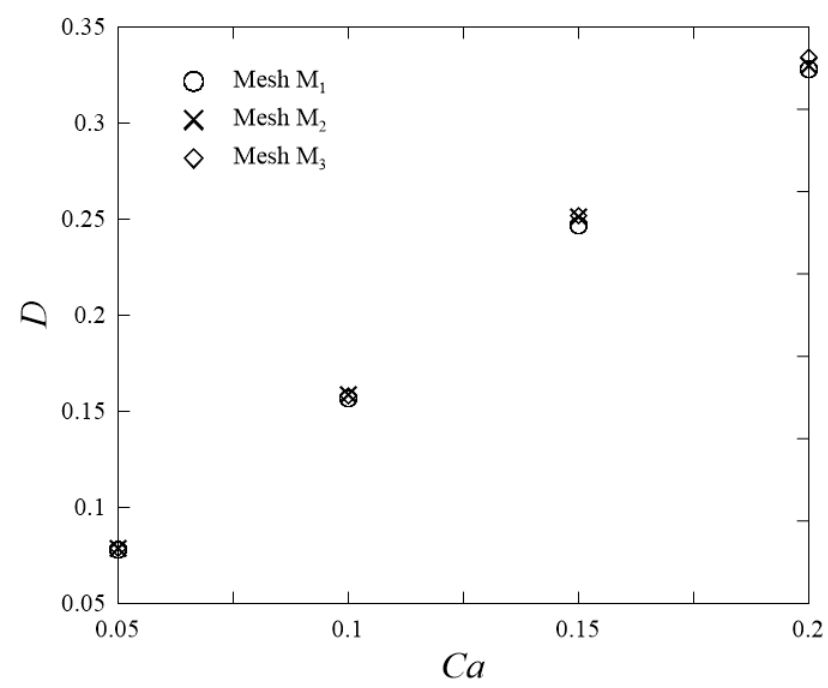

a)

Figure 5: Effect of the mesh resolution on the droplet deformation (a) and orientation angle (b) for the case $\xi=5.65$.

Table 2: Mesh refinement study: Values of the deformation and orientation angle for different capillary number and for three different levels of refinement. The last two columns show the relative difference percentage between the results for $\mathrm{M}_{1}$ and $\mathrm{M}_{2}$, and between $\mathrm{M}_{2}$ and $\mathrm{M}_{3}$ respectively.

\begin{tabular}{cccccc}
\hline \hline \multicolumn{7}{c}{ Deformation } \\
$C a$ & $\mathrm{M}_{1}$ & $\mathrm{M}_{2}$ & $\mathrm{M}_{3}$ & $\varepsilon_{12}[\%]$ & $\varepsilon_{23}[\%]$ \\
\hline 0.05 & 0.07811 & 0.07851 & 0.07870 & $\cong 1$ & $<1$ \\
0.1 & 0.15699 & 0.15852 & 0.15815 & $\cong 1$ & $<1$ \\
0.15 & 0.24669 & 0.25129 & 0.25206 & $\cong 1.5$ & $<1$ \\
0.2 & 0.32796 & 0.32976 & 0.33398 & $\cong 1$ & $\cong 1$ \\
\hline & & Orientation angle & & \\
$C a$ & $\mathrm{M}_{1}$ & $\mathrm{M}_{2}$ & $\mathrm{M}_{3}$ & $\varepsilon_{12}[\%]$ & $\varepsilon_{23}[\%]$ \\
\hline 0.05 & 61.291 & 60.74 & 60.731 & $\cong 1$ & $<<1$ \\
0.1 & 58.503 & 58.382 & 58.356 & $<1$ & $<<1$ \\
0.15 & 55.673 & 55.788 & 55.611 & $<1$ & $<1$ \\
0.2 & 52.909 & 52.699 & 52.557 & $<1$ & $<1$ \\
\hline \hline
\end{tabular}

Figure 6a shows the droplet deformation $D$ at steady state as a function of the capillary number for two different values of $\xi$ (corresponding to a magnetic field $\mathrm{H}=500 \mathrm{~A} / \mathrm{m} \quad(\xi=2.51)$ and $\mathrm{H}=750 \mathrm{~A} / \mathrm{m}(\xi=5.65))$ in addition to the reference case in the absence of a magnetic field $(\xi=0)$. 
Table 3: Constants $K$ and $C$ used in the fits of Figures $6 \mathrm{a}$ and $\mathrm{b}$ for different values of the parameter $\xi$

\begin{tabular}{cccc}
\hline \hline$\xi$ & 0 & 2.51 & 5.65 \\
$K$ & 1.03 & 1.2506 & 1.5347 \\
$C$ & $\infty$ & $\sim 0.1$ & 0.0575 \\
\hline \hline
\end{tabular}

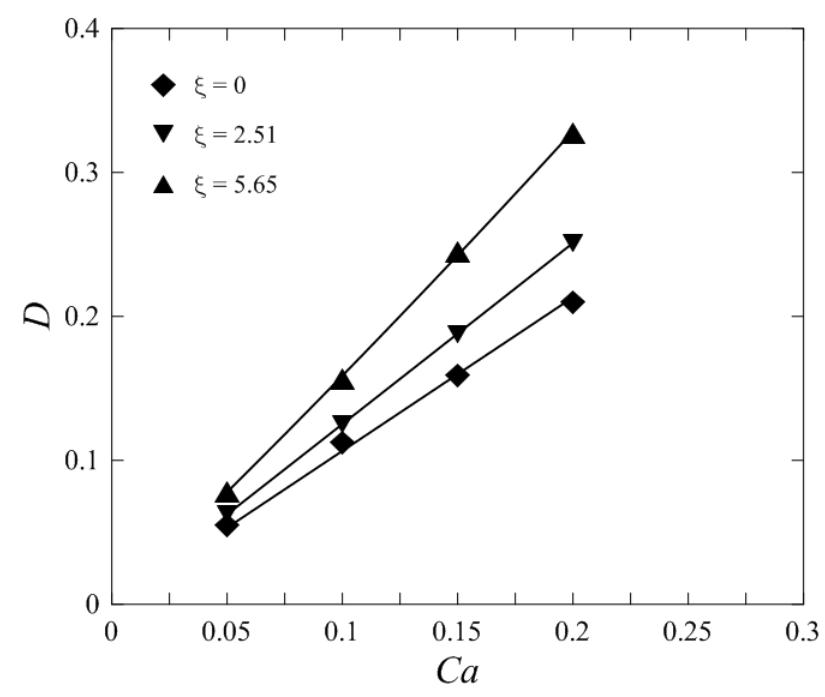

a)

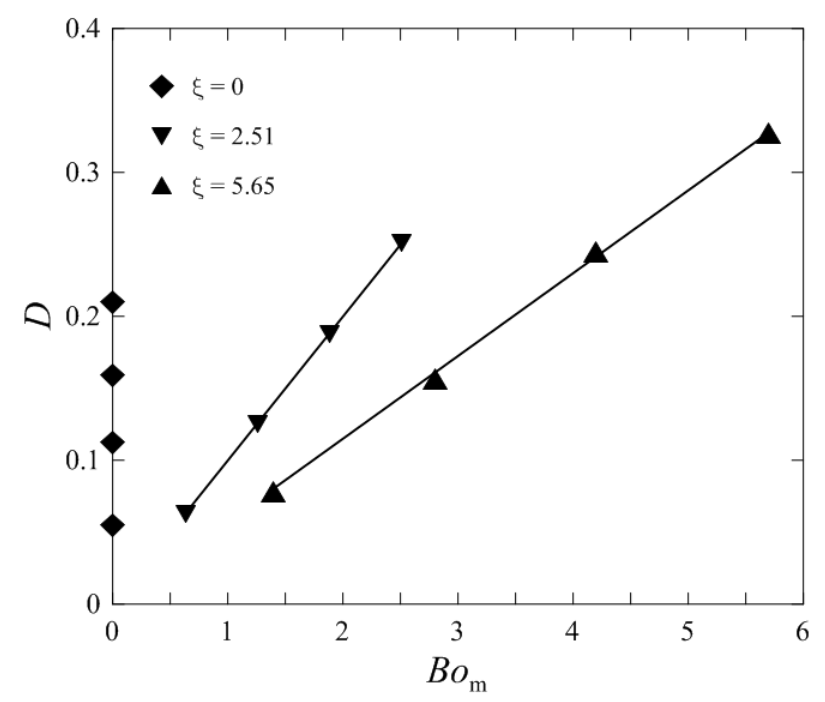

b)

Figure 6: Deformation of the ferrofluid droplet under the joint action of simple shear flow and magnetic field as function of the capillary number (a) and the magnetic Bond number (b) for different values of the parameter $\xi$.

As anticipated, the presence of the magnetic field enhances the deformation, which can become considerably larger than that observed for non-magnetizable fluids, especially for relatively high values of capillary number and parameter $\xi$. For a fixed value of the applied magnetic field, the deformation increases with the capillary number similarly to the case of a non-magnetizable droplet. Notably, also in the present configuration, for the specific values of $C a$ and $\xi$ considered, the deformation appears to be well interpolated by a linear fitting curve $D=K \cdot C a$ (curves depicted in the same figure).

As mentioned earlier, the system can be equivalently described by means of the parameter $\xi$ and one parameter to be chosen between the capillary number and the magnetic Bond number. Along these lines, in Fig. $6 \mathrm{~b}$ we illustrate the results from a different perspective by showing how the deformation changes as a function of the magnetic Bond number for different values of $\xi$. In this case, once again $D$ has been found to scale linearly with the magnetic Bond number following a law $D=C \cdot B o_{m}$, where $C=C(\xi)$ is a constant that decreases for increasing intensities of the applied magnetic field.

From a physical standpoint this reflects the obvious tendency of the droplet to remain spherical when the surface tension force becomes dominant with respect to both the viscous and magnetic forces. The values of the constants for the fitting laws for both the cases mentioned above are listed in Table 3. 
The effect of the magnetic field on the orientation angle $\varphi$ (cf. definition in Fig. 3a) is presented in Fig. 7a. Without the presence of magnetic force $(\xi=0)$, the angle decreases linearly from a maximum value of $\varphi \approx 42^{\circ}$, corresponding to $C a=0.05$, to a minimum value of $\varphi \approx 35^{\circ}$ for $C a=0.2$. As the magnetic force is increased, also the orientation angle becomes larger. This can be understood by considering that the droplet tends to align along the direction of the applied magnetic field (the magnetic force acts to stretch the droplet along its direction). Interestingly, however, although the orientation angle depends on $\xi$, as the reader will realize by inspecting the linear fitting lines depicted in Fig. 7a, the rate at which the angle decreases seems not to be affected by the competition between the magnetic force and the viscous force suggesting for the orientation angle the functional relationship $\varphi(C a, \xi)=K^{\prime} C a+f(\xi)$, where $K^{\prime}$ is a constant and $f(\xi)$ is a function that depends on the parameter $\xi$.

Fig. $7 \mathrm{~b}$ shows the superposition of two different drop shapes (for the case $\xi=2.51$ and $\xi=5.65$ ) for all the capillary numbers considered, showing the evident impact of the magnetic force on both deformation and orientation angle, with the differences becoming more evident for more intense magnetic fields.
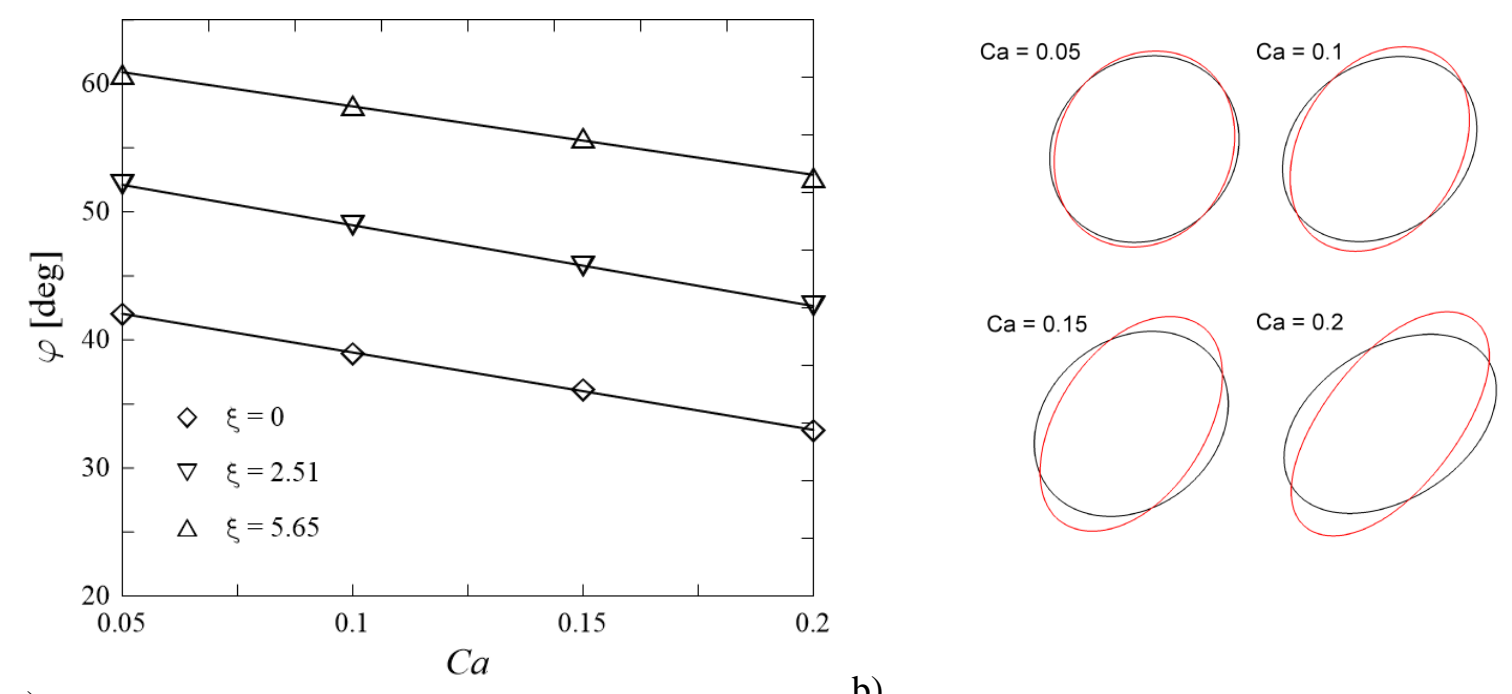

a)

b)

Figure 7: a) Orientation angle of the ferrofluid droplet under the joint action of simple shear flow and magnetic field as a function of the Capillary number for different intensities of the magnetic field. b) Comparison of the droplet shapes corresponding to $\xi=2.51$ (black line), 5.65 (red line) for different values of the capillary number. 


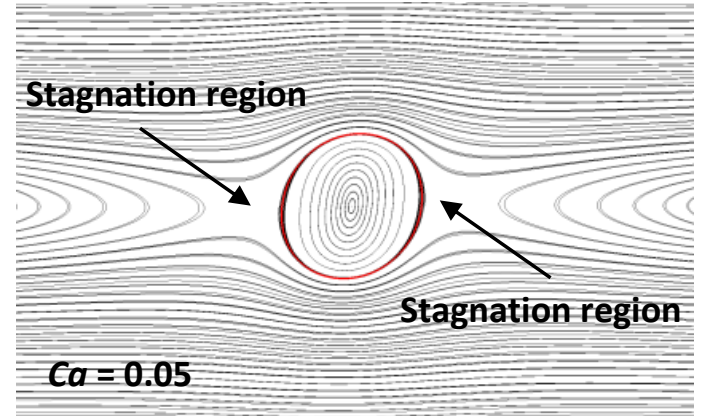

a1)

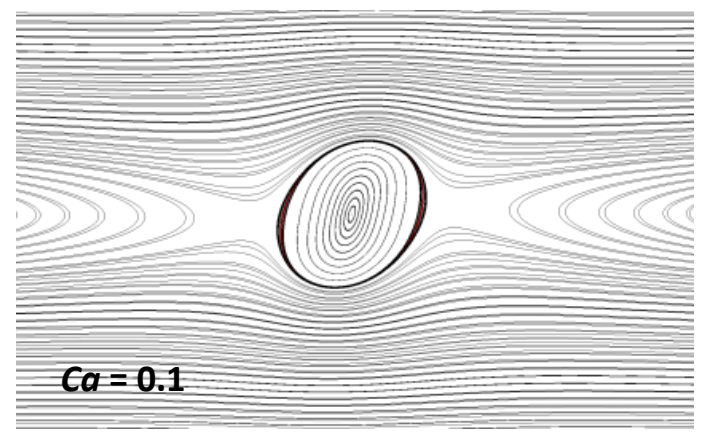

b1)

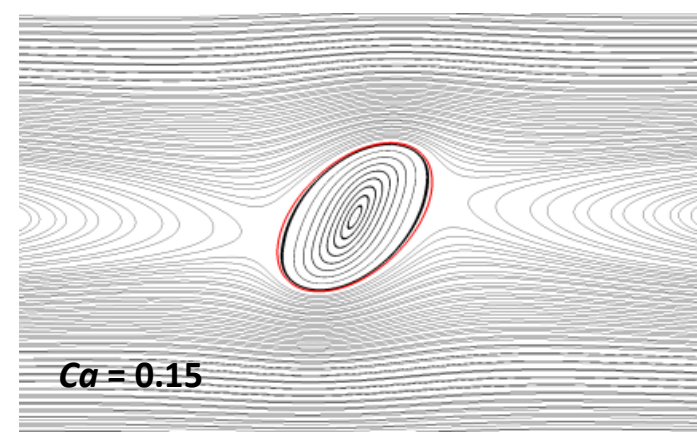

c1)

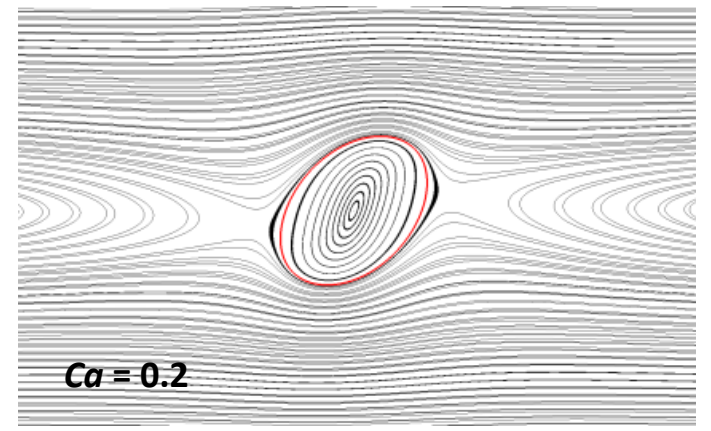

d1)

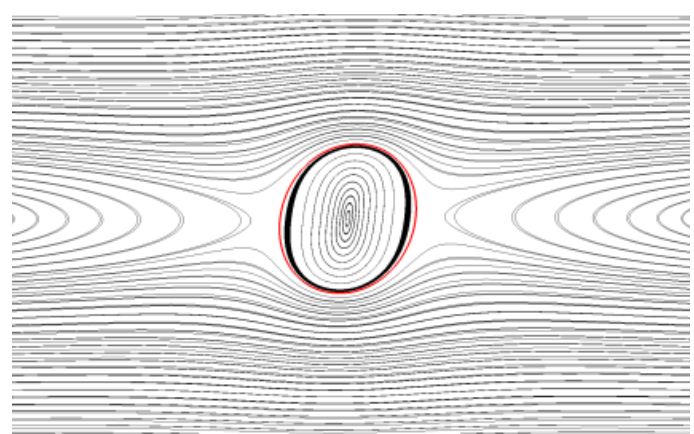

a2)

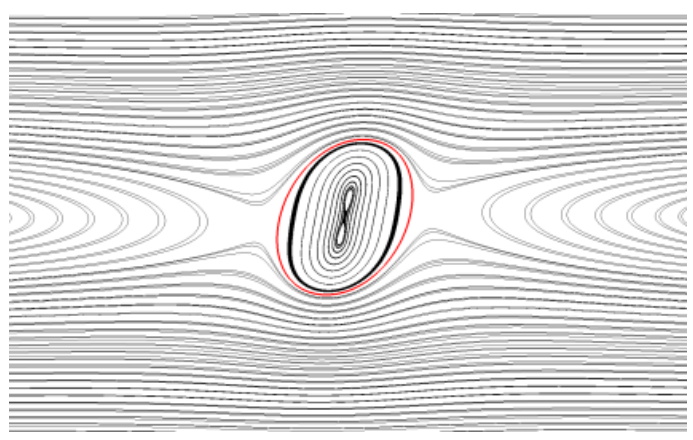

b2)

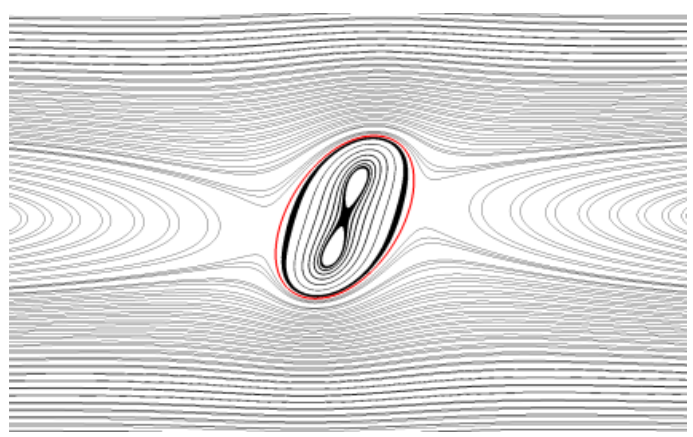

c2)

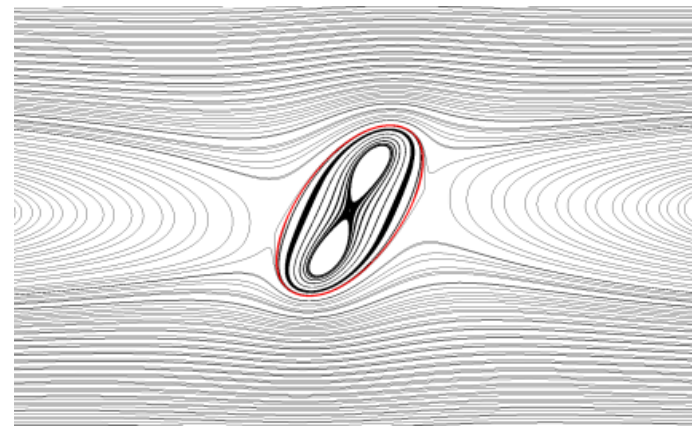

d2)

Figure 8: Streamlines in the region of the of the ferrofluid droplet under the joint action of simple shear flow and magnetic field obtained at the steady state for different values of the capillary number spanning from 0.05 to 0.2 for $\xi=2.51$ (left column, a1-d1) and for $\xi=5.65$ (right column, a2-d2) .

The behaviour in terms of streamlines is summarized in Fig. 8 (for $\xi=2.51$, (a1-d1 column), and $\xi=5.65$ (a2-d2 column) for all the capillary numbers considered. In the first case, the deformation 
remains relatively contained as the capillary number is increased. In particular, two relatively small stagnation regions can be noticed. These change their position, moving (in opposite direction with respect to the centreline of the domain) toward the lateral walls. For the highest value of $\xi$, however, the deformation increases with $\mathrm{Ca}$ at a larger rate and the more stretched droplet is more prone to become aligned with the magnetic field. The extension of the stagnation regions also increases and the flow patterns inside the droplet tend to develop multiple recirculating regions for the larger $\xi$, as seen in Figs. 8 (right column from b2 to d2).

\section{$\underline{3.3 \text { Droplet relaxation }}$}

In the present section, we finally look at the transient droplet relaxation dynamics that are produced when the applied magnetic field is suddenly switched off (evolutionary progress from the equilibrium configuration in the presence of the magnetic field, to the equilibrium state attained once the magnetic force is no longer applied). For simplicity, we assume that as the magnetic field is removed the magnetic force decays instantaneously. This allows us to focus directly on a situation where the relaxation process depends only on the competition between the capillary force and the viscous drag.

In order to capture the transient dynamics correctly, we set the time step, $\Delta \tau$, in such a way that $\Delta \tau<<t_{c}$, where $t_{c}=\eta_{m} R / \sigma$ represents a time scale characteristic of the flow under discussion. Figure 8 shows the temporal evolution of the relaxation process evaluated in terms of droplet deformation, $D$, normalized by its value in the final (equilibrium) state, $D_{0}$, as a function of the dimensionless time, $t / t_{c}$ for the cases $\xi=2.51$ (a), $\xi=5.65$ (b), and for two different capillary numbers $(C a=0.05, C a=0.1)$.

As evident in this figure, after experiencing an initial sudden contraction, the droplet undergoes a relatively weak shape modulation that is quickly damped by the effect of viscous stresses. Furthermore, we notice that for a fixed $\xi$, the dimensionless time required to reach the final steady state (relaxation time) displays a decreasing trend for increasing capillary numbers, which is in line with the role of the viscous force expected to become comparatively stronger irrespective to the capillary force when $\mathrm{Ca}$ is large.

By cross comparison of the left and right frames in Fig. 9, we also conclude that the relaxation time increases as the parameter $\xi$ becomes higher. Such behaviour might be ascribed to the different initial conditions (as $\xi$ become larger, the "distance" from the new equilibrium position increases with the magnetic field). In the same figure, we also show the fitting curves of equation $D / D_{0}=c_{1} e^{-c_{2} t^{*}}\left\{\sin \left(c_{3} t^{*}+c_{4}\right)\right\}$, where the $c_{1}, c_{2}, c_{3}$ and $c_{4}$ are constants and $t^{*} \equiv t / t_{c}$ has been introduced for convenience of notation, which resembles the decay process of a harmonic oscillator in the presence of viscous damping. 
Notably, the fitting law is more accurate for $\xi=2.51$, which corresponds to initial conditions closer to the final steady state configuration (smaller amplitude of the deformation).
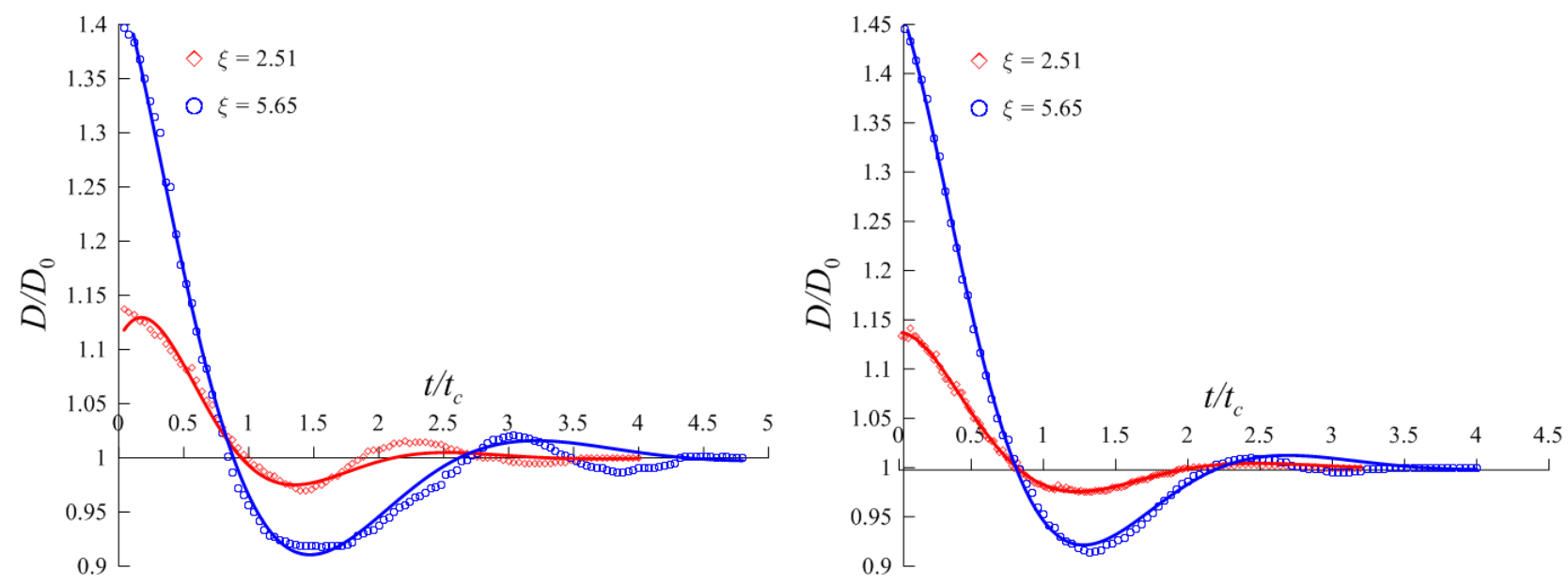

a)

b)

Figure 9: Normalized droplet deformation temporal evolution for the case when the applied magnetic field is suddenly switched off: (a) $C a=0.05$, (b) $C a=0.1$.

\section{Conclusions}

We have investigated the effect of an imposed constant magnetic field on the shear-induced deformation of a two-dimensional ferrofluid droplet in the Stokes flow regime. Several flow conditions have been explored by varying parametrically the capillary number and the parameter $\xi$ while maintaining the magnetic susceptibility of the ferrofluid droplet unchanged.

We have found that the presence of the magnetic field has a considerable influence on the deformation of the droplet, which can increase significantly. The orientation angle is also affected by the presence of the magnetic field. The rate with which it changes due to an increase in $\mathrm{Ca}$, however, seems to be only slightly dependent on the entity of the applied magnetic field.

The relaxation process from equilibrium conditions with the magnetic force to equilibrium in absence of such constraint has been also considered. We have found that the droplet deformation tends to the new steady state following a harmonic exponential decaying law formally resembling the mechanism of relaxation of a damped oscillator. In line with physical arguments, such law fits the data more correctly when the initial conditions are such that the droplet shape is less deformed (smaller capillary numbers). The relaxation time depends on both on the capillary number and on the parameter $\xi$. In particular, for a fixed $\xi$, the time required to achieve the new equilibrium condition decreases as the capillary number is increased due to the increase strength of the viscous damping forces with respect to the recalling capillary force. Furthermore, on increasing the parameter $\xi$, the relaxation process 
becomes longer due to the increased initial droplet deformation irrespective to the final condition in the absence of magnetic effects.

For future studies three-dimensional configurations will be examined. The investigation will be also devoted to consider the impact of different susceptibilities and viscosity ratios on the final deformation, possibly covering the range of parameters in which the end-pinching of the droplet occurs.

\section{References}

[-] Abgrall P and Gué A.M., (2007), Lab-on-chip technologies: making a microfluidic network and coupling it into a complete microsystem-a review, J. Micromech. Microeng. 17: R15-R49

[-] Afkhami, S., Tyler, A. J., Renardy, Y., Renardy, M., Pierre, T., G., St., Woodward, R. C. and Riffle, J., S., (2010), Deformation of a hydrophobic ferrofluid droplet suspended in a viscous medium under uniform magnetic fields, J. Fluid Mech, 663, 358-384.

[-] Albadawi A., Donoghue D. B., Robinson A. J., Murray D. B., Delauré Y. M. C. (2013), Influence of surface tension implementation in volume of fluid and coupled Volume of Fluid with Level Set methods for bubble growth and detachment, Int. J. Multiphase Flows, 53, 11-28.

[-] Bacri J. C. and Salin D., (1982), Instability of ferrofluid magnetic drops under magnetic field. Journal de Physique Lettres 43.17: 649-654.

[-] Barai N., and Mandal N., (2016), Breakup modes of fluid drops in confined shear flows, Physics of Fluids, 28(7), 073302.

[-] Behjatian, A. and Esmaeeli, A., (2013), Electrodynamics of a compound drop, Physical Review E $88,033012$.

[-] Berberović, van Hinsberg N. P., Jarkirlić S., Roisman L. V., Tropea C., (2009), Drop impact onto a liquid layer of finite thickness: Dynamics of the cavity evolution, Phys. Rev. E, 79, 036306.

[-] Bławzdziewicz, J., V. Cristini, and M. Loewenberg, (2003) 'Multiple stationary states for deformable drops in linear stokes flows, Phys. Fluids 15, L37-L40.

[-] Brackbill J.U., Kothe D.B., Zemach C., (1992), A Continuum Method for Modeling Surface Tension, J. Comput. Phys., 100 (2): 335-354.

[-] Capobianchi, P., Lappa, M., Oliveira, M.S. N., (2017), Implementation of a Flexible and Modular Multiphase Framework for the Analysis of Surface-Tension-Driven Flows Based on a LS-VOF Approach, in Selected papers of the 11th OpenFOAM Workshop, J.M.Nóbrega and H. Jasak (Editors), Springer Nature, 2018, ISBN - 978-3-319-60845-7. 
[-] Capobianchi, P., Lappa, M., Oliveira, M.S. N., (2017), Walls and Domain Shape Effects on the Thermal Marangoni Migration of Three-dimensional Droplets, Phys. Fluids 29, 112102.

[-] Chen Y.J., Nagamine Y. and Yoshikawa K., (2009), Self-propelled motion of a droplet induced by Marangoni-driven spreading, Phys. Rev. E 80, 016303

[-] Chen, Ching-Yao, and Z-Y. Cheng., (2008), An experimental study on Rosensweig instability of a ferrofluid droplet, Phys. Fluids 20.5, 054105.

[-] Cristini, V., Guido, S., Alfani, A., Bławzdziewicz, J., and Loewenberg, M., (2003), Drop breakup and fragment size distribution in shear flow, Journal of Rheology 47, 1283 (2003);

[-] Deshpande S. S., Anumolu L., and Trujillo M., (2012), Evaluating the performance of the twophase flow solver interFoam, Computational Science and Discovery, 5, 014016.

[-] Dickstein A. J., Erramilli S., Goldstein R. E., Jackson D. P., and Langer S. A., (1993), Labyrinthine Pattern Formation in Magnetic Fluids, Science, 261: 1012-1015

[-] Drozdova V. I., Skrobotova T. V., and Chekanov V. V. (1979), Experimental study of the hydrostatics characterizing the interphase boundary in a ferrofluid, Magnetohydrodynamics, 15(1), 12-14.

[-] Hong R., Ren Z., Zhang S., Ding J., Li H., (2007), Numerical simulation and experimental verification of silicone oil flow over magnetic fluid under applied magnetic field, China Particuology 5: 93-102.

[-] Ioannou, N., Liu, H., Zhang Y.H., (2016), Droplet Dynamics in Confinement, Journal of Computational Science, Volume 17, Part 2, pp 463-474.

[-] Ivanov I.B. and Kralchevsky P.A., (1997), Stability of Emulsions under Equilibrium and Dynamics conditions, Colloids \& Surfaces A, 128: 155-175.

[-] Ki H., (2010), Level Set Method for Two-Phase Incompressible Flows Under Magnetic Fields, Computer Physics Comm., 181, 999-1007.

[-] Klostermann, J., Schaake, K., and Schwarze, R., (2012), Numerical simulation of a single rising bubble by VOF with surface compression, Int. J. Numer. Meth. Fluids, 71: 960-982.

[-] Korlie M. S., Mukherjee A., Nita B. G., Stevens J. G., Trubatch A. D., and Yecko P. (2008). Modeling bubbles and droplets in magnetic fluids. Journal of Physics: Condensed Matter, 20(20), 204143.

[-] Lamb, H., Hydrodynamics, 6th ed. (Cambridge University Press, Cambridge, 1932).

[-] Lappa M., (2005), Coalescence and non-coalescence phenomena in multi-material problems and dispersed multiphase flows: Part 2, a critical review of CFD approaches, Fluid Dynamics \& Materials Processing, 1(3): 213-234. 
[-] Lavrova, O., Matthies, G., Polevikov, V., and Tobiska, L., (2004), Numerical Modeling of the Equilibrium Shapes of a Ferrofluid Drop in an External Magnetic Field, Proc. in Appl. Mathematics and Mechanics, 4, 704-705.

[-] Morris C.J. and Parviz B.A., (2006), Self-assembly and characterization of Marangoni microfluidic actuators, J. Micromech. Microeng. 16: 972-980.

[-] Nelson B.J., Kaliakatsos I.K., Abbott J.J., (2010), Microrobots for minimally invasive medicine, Annu Rev Biomed Eng., 12:55-85.

[-] Nguyen N.T., (2011), Micro-magnetofluidics: interaction between magnetism and fluid flow on the microscale, Microfluidics and Nanofluidics, 12(1-4): 1-16

[-] Rallison, J. M., (1984), The deformation of small viscous drops and bubbles in shear, Annu. Rev. Fluid Mech. 16, 45-66.

[-] Rhie M. C, Chow L. W., (1982), A numerical study of the turbulent fow past an isolated airfoil with trailing edge separation, AIAA-82-0998.

[-] Rosensweig R. E., (1985), Ferrohydrodynamics, Cambridge University Press, Cambridge, 1985.

[-] Roths T., Friedrich C., Marth M., Honerkamp J., (2002), Dynamics and rheology of the morphology of immiscible polymer blends - on modeling and simulation, Journal Rheologica Acta, 41(3): 211-222.

[-] Sherwood J. D., (1988), Breakup of fluid droplets in electric and magnetic fields. J. Fluid Mech., $188,133-146$.

[-] Shi D., Bi Q., and Zhou R. (2014), Numerical simulation of a falling ferrofluid droplet in a uniform magnetic field by the VOSET method, Numer. Heat Transfer, Part A: Applications, 66(2), 144-164.

[-] Stone H. A., (1994), Dynamics of drop deformation and breakup in viscous fluids, Annual Review of Fluid Mechanics, 26(1), 65-102.

[-] Stone H. A., Lister J. R., and Brenner M. P., (1999), Drops with conical ends in electric and magnetic fields. In Proceedings of the Royal Society of London A: Mathematical, Physical and Engineering Sciences (Vol. 455, No. 1981, pp. 329-347). The Royal Society.

[-] Son, G., Hur, N., 2002. A coupled level set and volume-of-fluid method for the buoyancy-driven motion of fluid particles. Numer. Heat Transfer, Part B: Fundam. 42, 523-542.

[-] Sun, D., Tao, W., 2010. A coupled volume-of-fluid and level set (voset) method for computing incompressible two-phase flows. Int. J. Heat Mass Transfer 53, 645-655.

[-] Sussman M., Smereka P., Osher S., (1994), A Level Set Approach for Computing Solutions to Incompressible Two-Phase Flow, J. Comput, Physics, 114(1),146-159.

[-] Tran N., and Webster T. J. (2010). Magnetic nanoparticles: biomedical applications and challenges. Journal of Materials Chemistry, 20(40), 8760-8767. 
[-] Tucker C. L., and P. Moldenaers, (2002), Microstructural evolution in polymer blends, Annu. Rev. Fluid Mech. 34, 177-210.

[-] Xia H., Wang J., Tian Y., Chen Q.-D., Du X.-B., Zhang Y.-L., He Y. and Sun H.-B., (2010), Ferrofluids for Fabrication of Remotely Controllable Micro-Nanomachines by Two-Photon Polymerization, Adv. Mater., 22: 3204-3207.

[-] Yamamoto T., Okano Y., Dost S., (2016), Validation of the S-CLSVOF method with the densityscaled balanced continuum surface force model in multiphase systems coupled with thermocapillary flows, Int. J. Numer. Meth. Fluids, 83(3): 223-244.

[-] Yue P., Feng J. J., Liu C., Shen J., (2004), A diffuse-interface method for simulating two-phase flows of complex fluids, J. Fluid Mech. pp 293-317.

[-] Zander R., Dittman M., and Schneider G.M., (1990), Dissipative structures in demixing binary liquid systems, Z. Naturforsch, 45a: 1309-1316.

[-] Zhou H. and Pozrikidis C., (1993), The flow of suspensions in channels: Single files of drops, Phys. Fluids, 5(2.), 311-324.

[-] Zhu G.-P., Nguyen N.-T., Ramanujan R. V., and Huang X.-Y., (2011), Nonlinear Deformation of a Ferrofluid Droplet in a Uniform Magnetic Field, Langmuir, 27, 14834-14841. 